\title{
Saint-Andoche et Saint-Jean-le-Grand : des religieuses à Autun au Moyen Âge
}

Thèse de doctorat de l'université Paris 1 Panthéon Sorbonne, sous la direction de Michel Parisse, 10 décembre 2009

Nathalie Verpeaux

\section{(2) OpenEdition}

Édition électronique

URL : https://journals.openedition.org/cem/11598

DOI : 10.4000/cem.11598

ISSN : 1954-3093

Éditeur

Centre d'études médiévales Saint-Germain d'Auxerre

Référence électronique

Nathalie Verpeaux, "Saint-Andoche et Saint-Jean-le-Grand : des religieuses à Autun au Moyen Âge », Bulletin du centre d'études médiévales d'Auxerre / BUCEMA [En ligne], 14 | 2010, mis en ligne le 12 octobre 2010, consulté le 22 septembre 2022. URL : http://journals.openedition.org/cem/11598 ; DOI : https:// doi.org/10.4000/cem.11598

Ce document a été généré automatiquement le 22 septembre 2022.

\section{() $(\otimes \otimes \odot$}

Creative Commons - Attribution - Pas d'Utilisation Commerciale - Partage dans les Mêmes Conditions 4.0 International - CC BY-NC-SA 4.0

https://creativecommons.org/licenses/by-nc-sa/4.0/ 


\section{Saint-Andoche et Saint-Jean-le- Grand : des religieuses à Autun au Moyen Âge}

Thèse de doctorat de l'université Paris 1 Panthéon Sorbonne, sous la direction de Michel Parisse, 10 décembre 2009

\section{Nathalie Verpeaux}

1 Autun, siège d'un vaste et puissant évêché dès le début du Ive siècle au plus tard, a été appelée par Louis XII la cité " aux biaux clochiers " ${ }^{1}$. Il est vrai que la cité comptait, à l'intérieur de la vaste enceinte antique, deux cathédrales, sept églises paroissiales, dont une érigée en collégiale $a u x^{e}$ siècle, et deux abbayes de femmes, ainsi que, à l'extérieur des remparts, deux prieurés et une abbaye d'hommes. Les gens d'Église étaient donc très présents dans la ville et une telle coexistence de deux abbayes féminines - Saint-Andoche et Saint-Jean-le-Grand en l'occurrence -, situées à moins d'un kilomètre l'une de l'autre, durant plus de huit siècles, était assez rare pour justifier une étude monographique.

2 C'est à comprendre le fonctionnement de ces deux abbayes de femmes de l'ordre de Saint-Benoît, leurs points communs et leurs différences, mais aussi leur intégration dans la ville et la société que s'est attaché mon travail ${ }^{2}$.

3 Cette étude a été possible grâce à la conservation de fonds d'archives particulièrement importants pour des abbayes féminines - plus de mille liasses pour les périodes médiévale et moderne aux seules Archives départementales de Saône-et-Loire, sans compter les quelques liasses conservées à Dijon ou Autun notamment. En revanche, aucun bâti médiéval, hormis le chevet de l'église paroissiale dépendant d'une des deux abbayes, n'est encore en place, les deux abbayes ayant été entièrement reconstruites aux XVII ${ }^{e}$ et $\mathrm{XVIII}^{\mathrm{e}}$ siècles et leurs églises au XIXe siècle. Toute étude archéologique est également impossible en raison du très grand nombre de constructions actuelles sur les sites de ces abbayes. Malgré la conservation de plans du XVI e siècle, à la fois très précis et généralement fiables, et de quelques gravures des $\mathrm{XVII}^{\mathrm{e}}$ et $\mathrm{XVIII}$ e siècles, l'emprise physique des abbayes dans la ville est difficile à déterminer. 
4 L'origine de ces deux abbayes carolingiennes remonte en fait au vi siècle, à une abbaye de femmes dédiée à sainte Marie et attestée dès 589 et à un xenodochium fondé quelques années plus tard par l'évêque Syagrius et la reine Brunehaut, en même temps qu'une église pour des clercs dédiée à saint Martin, église transformée par la suite en abbaye éponyme. Se pose ici la question de la localisation exacte de l'abbaye SainteMarie et du xenodochium. Ce dernier, ayant pour mission d'accueillir des pèlerins, les pauvres et les malades, devait se situer à proximité à la fois du principal noyau de peuplement de la cité et d'une porte d'accès à celle-ci, sans doute au niveau de la porte de Clermont, là où fut fondée plus tard l'abbaye Saint-Andoche, comme le rapporte la tradition d'ailleurs. Quant à l'abbaye Sainte-Marie, antérieure, rien ne permet de la localiser à l'intérieur de la vaste zone délimitée par les remparts anciens. La tradition la situe à l'emplacement plus tardif de Saint-Jean-le-Grand, ce qui n'est pas illogique : cette abbaye féminine aurait été refondée sur les lieux d'une ancienne abbaye de femmes, là où restaient peut-être quelques structures après une disparition liée aux raids sarrasins. Toutefois, l'hypothèse selon laquelle elle aurait été située à proximité de la porte de Clermont et aurait été associée, à partir des dernières années du $\mathrm{VI}^{\mathrm{e}}$ siècle, au xenodochium nouvellement fondé, ne peut pas être rejetée non plus.

Quoi qu'il en soit, ces deux établissements anciens ont disparu dans la tourmente du premier tiers du VIII siècle, lors de l'incendie de la cité par les Sarrasins. Saint-Andoche fut, semble-t-il, fondée aussitôt après par Décie, sœur du fondateur de Flavigny, et est bien attestée au IX ${ }^{\mathrm{e}}$ siècle à la fois par les textes et l'archéologie. À la première catégorie appartiennent un privilège épiscopal et trois diplômes royaux, même si cette documentation soulève des problèmes diplomatiques d'authenticité et de datation. Une mystérieuse crypte, qui a été à l'origine de nombreuses hypothèses, daterait en fait de la fin du $\mathrm{IX}^{\mathrm{e}}$ ou du début $\mathrm{x}^{\mathrm{e}}$ siècle et a sans doute eu une fonction liturgique en liaison avec l'abbatiale préexistant à celle $\mathrm{du} \mathrm{xv}^{\mathrm{e}}$ siècle, d'après l'étude qu'en a faite Christian Sapin ${ }^{3}$. Sont conservés, d'autre part, sept deniers de Charles le Chauve postérieurs à l'édit de Pîtres, frappés au nom de Saint-Andoche d'Autun ${ }^{4}$, peut-être par délégation du droit monétaire du chapitre cathédral. La (re)fondation de Saint-Jean-le-Grand est totalement inconnue, mais l'abbaye jouissait déjà, sans aucun doute, d'un grand prestige, quand, vers 990, Gerberge, descendante des seigneurs de Vienne, mère d'Odilon alors le plus proche conseiller de l'abbé de Cluny Maïeul, y prit le voile suite à son veuvage.

6 Le développement des deux abbayes par la suite et jusqu'au XIV siècle reste méconnu. Toutefois, des bourgs se créent autour de ces deux centres religieux et dans leur dépendance économique et juridique notamment. Les deux communautés devant à la fois assurer la cura animarum de ces populations et préserver leur propre tranquillité, durent alors faire bâtir deux églises paroissiales, dont l'existence est attestée au milieu du XIII ${ }^{\mathrm{e}}$ siècle par leur mention dans des testaments ; l'étude du chevet de SaintPierre Saint-Andoche a toutefois permis à Marine Revenu de le dater du second quart du XII $^{e}$ siècle ${ }^{5}$. Il est intéressant de noter que les deux communautés n'ont pas fait construire les églises paroissiales qui dépendaient d'elles dans les mêmes conditions : Saint-Pierre-Saint-Andoche est située à proximité de l'abbaye, mais clairement en dehors de la clôture, alors que Saint-Jean-du-Bourg - dont le patronage est identique à celui de l'abbatiale - est située à l'intérieur de la clôture, parallèle à l'abbatiale dont elle n'est séparée que par une ancienne tour carrée, qui sert de clocher commun aux deux églises. Cette configuration très rare, en particulier dans une abbaye de femmes, 
pose aujourd'hui des problèmes d'identification et de localisation exacte de ces deux églises, qui n'apparaissent d'ailleurs pas clairement distinctes sur les plans du $\mathrm{XVI}^{\mathrm{e}}$ siècle.

7 Grâce à ces églises paroissiales et aux confréries qui s'y sont installées, mais aussi grâce aux châtelets à l'intérieur desquels les religieuses étaient l'unique seigneur et l'unique pouvoir, ainsi que grâce à leurs nombreuses possessions de terres, terrains, maisons et bâtiments, les dames de Saint-Andoche et de Saint-Jean étaient bien intégrées dans la société autunoise. C'est d'ailleurs aussi ce que montrent les obituaires de SaintAndoche, qui attestent de fondations d'anniversaires par des petites gens du quartier Saint-Andoche tout autant que par certaines des puissantes familles autunoises; les religieuses participaient aussi régulièrement à des cortèges funéraires et, plusieurs fois par an, aux grandes processions religieuses.

8 Pourtant le recrutement autunois est faible, hormis au $\mathrm{Xv}^{\mathrm{e}}$ siècle, où, notamment à Saint-Andoche, quelques religieuses étaient issues de familles parmi les plus en vue de la cité. En effet, les religieuses de ces deux abbayes urbaines appartenaient essentiellement à des familles de l'aristocratie rurale bourguignonne; elles venaient de seigneuries proches d'Autun - moins de 20 kilomètres autour de la cité -, mais aussi de seigneuries beaucoup plus lointaines - plus de 60 , voire plus de 100 kilomètres autour d'Autun. Quelques filles de familles extrêmement prestigieuses, alliées parfois à la maison ducale, se sont aussi retirées à Autun, souvent pour y occuper les plus hautes fonctions, notamment l'abbatiat. Grâce aux liens conservés avec leur famille et du fait des alliances nouées par celles-ci, qui donnent naissance à des groupes familiaux élargis au sein des abbayes, se créent en effet des réseaux qui favorisent la carrière de certaines religieuses. De telles stratégies font apparaître une hiérarchie entre les établissements féminins: les prieurés ruraux proches d'Autun - Saint-Georges-desBois et Pierre-Cervau, mais aussi Saint-Julien-sur-Dheune et Champchanoux - sont moins prisés que les deux abbayes urbaines, elles-mêmes moins recherchées que les grandes abbayes lorraines de dames nobles, notamment Remiremont; les mouvements de religieuses, notamment, suggèrent aussi que Saint-Andoche était un établissement un peu plus convoité que Saint-Jean aux XIV et XV ${ }^{e}$ siècles.

9 Saint-Andoche et Saint-Jean-le-Grand étaient en effet parfaitement intégrées à la société bourguignonne, notamment grâce à leurs nombreux domaines et seigneuries parfois éloignés d'Autun: Saint-Jean était possessionnée jusque dans le comté de Bourgogne autour de Colonne, Saint-Andoche jusque dans le Montbardois autour de Senailly et en Nivernais autour de Dorne, ce qui faisait connaître les abbayes éduennes en ces lieux lointains. En effet, les religieuses y étaient physiquement présentes pour surveiller la bonne gestion de ces domaines: si d'éventuels prieurés occupés par des religieuses de Saint-Andoche ou de Saint-Jean ont disparu en tant que tels au XIII siècle au plus tard, les religieuses, représentées sur place par des convers ou par des officiers qui pouvaient porter le titre de maire, de prévôt ou de doyen, avaient mis en place un système de droit de gîte qui leur permettait de se rendre personnellement sur place. L'exemple de la maison seigneuriale de Senailly, dont un plan est conservé pour le début $\mathrm{du} \mathrm{xVIII}^{\mathrm{e}}$ siècle ${ }^{6}$, montre d'ailleurs une chapelle dont le chœur était anormalement allongé, ce qui y atteste de la possibilité de réunion de quelques religieuses pour les offices quotidiens. Les comptes mentionnent également de nombreux déplacements, notamment des abbesses, dans les différents domaines appartenant aux religieuses (fig. 1). 


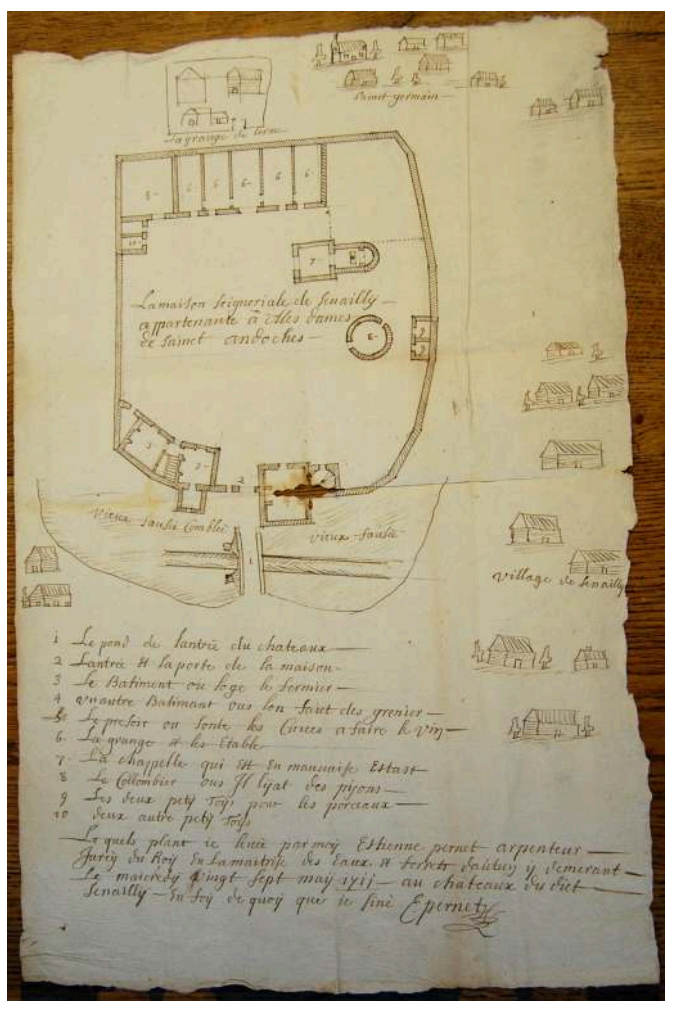

Fig. 1 - La maison seigneuriale de Senailly appartenant aux dames de Saint-Andoche.

10 Une étude d'histoire religieuse, monographie de deux abbayes féminines, amène donc à étudier une ville et une région, à en favoriser la connaissance sous certains aspects originaux, d'autant qu'une étude familiale et biographique a été menée sur environ trois cents religieuses et sept cents proches des abbayes - officiers des abbayes, mais aussi fondateurs et bénéficiaires d'anniversaires.

\section{NOTES}

1. H. ABORD, Histoire de la Réforme et de la Ligue dans la ville d'Autun, 1855-1886, Autun/Paris, t. 1 , p. 40, n. 1 .

2. Ce dernier point sera plus particulièrement développé ici, une présentation couvrant l'ensemble des facettes de ce travail de façon plus homogène devant paraître dans les Annales de Bourgogne.

3. C. SAPIN, La Bourgogne préromane, Paris, 1986, p. 37-39 ; ID., "Le monastère Saint-Andoche », in Autun, Augustodunum, Autun, 1987, p. 382-384.

4. M. Prou, Les monnaies carolingiennes, Paris, 1896, pl. XIV, n.600-602; J. LAFAURIE, « Numismatique des Carolingiens aux Capétiens », Cahiers de civilisation médiévale, 13 (1970), p. 117-137 ; Cl. M. HAERTLE, Karolingische Münzfunde aus dem 9. Jahrhundert, Cologne, Weimar/ Vienne, 1997, p. 970, n 624/001; G. DEPEYROT, Le numéraire carolingien corpus des monnaies, Paris, 1993, p. 19 (2e éd. augmentée, Wetteren/Paris, 1998, p. 122, nº 92).

5. M. REVENU, L'église de Saint-Pierre-Saint-Andoche à Autun, mémoire de maîtrise d'archéologie, université de Paris 10, p. 72-76. 
6. Archives départementales de la Côte-d'or, 74 H 6 (1711).

INDEX

Index géographique : France/Autun

Mots-clés : religieuse 